\title{
Study on the Humachine Intelligent System and Its Application
}

\author{
Lu Yongxiang*, Yang Canjun**, Chen Ying** \\ * Chinese Academy of Sciences \\ 52 Sanlihe, Beijing, 100864 P. R. China \\ **The State Key Laboratory for Fluid Power Transmission and Control \\ Zhejiang University \\ 20 Zheda Road, Hangzhou, 310027 P. R. China \\ (E-mail: ycj(i) sip.zju.edu.cn, http://sip.zju.edu.cn)
}

\begin{abstract}
It is a brand new method for setting up the intelligent system in the paper, which aimed at the improvement of intelligent technology at present. It is named humachine intelligent system (HIS). In the paper, the authors discuss the architecture of humachine intelligent system, and put forward three kinds of humachine cooperation methods such as "Human Master/Machine Slave (HM/MS)", "Machine Master/Human Slave (MM/HM)" and "Human Machine Collaboration (HMC)" to accomplish the humachine intelligence. Automobilism is a very complicated manipulation, in order to improve the automobilism security. The authors put forward an intelligent driving method based on the idea of humachine system and carry out the simulation experimentation by an automobile simulator., which indicates that the humachine intelligent system has prodigious feasibility and superiority.
\end{abstract}

\section{KEY WORDS}

Humachine system, Artificial Intelligence, Man-machine System

\section{INTRODUCTION}

At present, there are many methods for the implement of the intelligent system, but most of them have their diverse disadvantages. For example, the artificial intelligence which is based on symbol mechanism obtains the result from modeling the external world, but it is very difficult to setup the entireness right model. The another one is neural network method which is based on link mechanism obtains results from simulating human brain, but up to now people have not been able to understand the human brain mechanism very well yet.
The third method to simulate human intelligence is to combine artificial intelligence with neural network. Although this method has overcome aforementioned shortages, it can only simulate logical thinking not imaginary thinking. Fuzzy theory is another simulating intelligence methods, but it must setup too many fuzzy rules in advance to solve the complex questions well.

Therefore, many scholars put forward the idea of setting up human machine integrated intelligent system. The humachine intelligent system is one new idea of them. 


\section{HIS Modeling}

How to make man play a role to the full in the system and build up a new cooperation relationship between man and machine to setup the low cost, high benefit, high performance intelligent system? The author have put forward the theory, architecture and technology of humachine intelligent system before[1][2][3]. By the HIS we mean that the system consists of human and machine together just as equal. They do their own fittest work respectively, they help one another and make up one another's deficiencies. They sense, perceive, think, make decision and work together as a whole. They understand, restrict and supervise one another. Thus the superintelligent system, as to speak, is formed by breaking through the concept of traditional intelligent system.

Under this definition, The humachine intelligent system is meta-synthesized in perceptive, thinking and executive levels. The point of HIS is emphasizing the important of man in loop. The teamwork relation has been defined between man and machine, human-machine coupling can be implemented with the hypermedia, and the humanmachine synergy has been realized.

Therefore, the model of HIS can be modified as figure 1 based on HIS theory mentioned above.

greater, man or machine. We care if the result is satisfied.

\section{REALIZATION TACTICS OF HIS}

The key to realize the humachine intelligent system is how to distribute tasks to human and machine rationally and how to make them cooperate well. Under the complex conditions, the different tactics are adopted to solve special problems.

\section{Human Master/Machine Slaver}

There are many systems which are ill-structured, nonlinear or which are very important to people security. Almost all of these questions have been solved by people
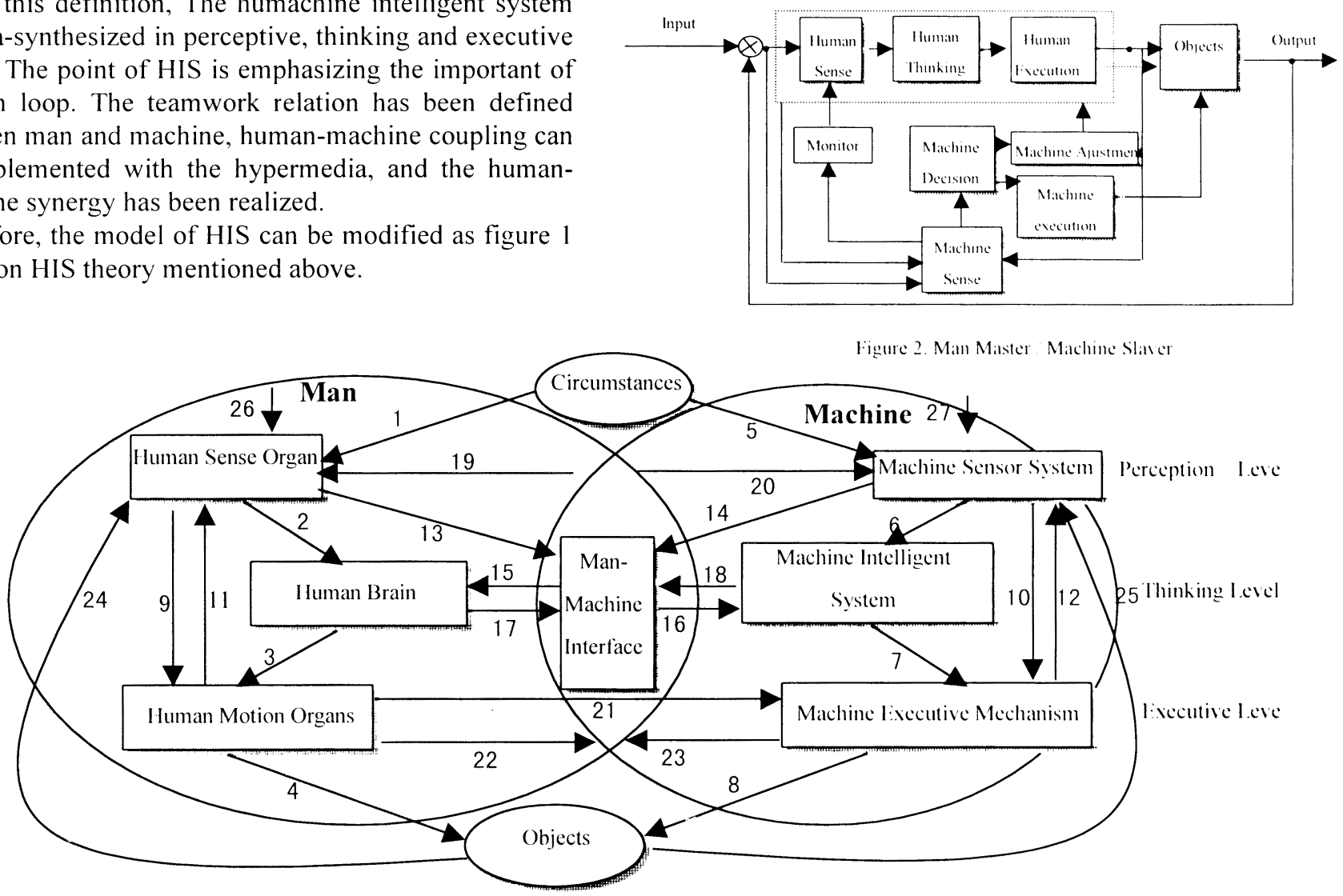

Figure 1. The humachined intelligent system general model

Figure 1 show that the humachine intelligent system consists of sense, thinking and executive three levels. Arrows ranging from 1 to 4 show that the task is manipulated by human alone, arrows ranging from 5 to 8 mean that the task is accomplished by machine only. Other arrows here in the figure show that man and machine accomplish work together in the humachine intelligent system. We do not care about or it is not important to know about which influenced the result alone in order to make the systems operating well and prevent operators from dangers, such as automobile driving.

Under such conditions, the machine intelligence can been added in this system, thus the total performance of the systems can be improved and the human's labor intensity can be reduced. This tactic is named as "Human master/Machine slaver" tactic, whose structure can be described as figure 2. 
Under this tactic, human can accomplish the task, but the circumstance information can not be sensed by human completely because of human physiology and psychological limitation. There are some obvious deviations and errors appear sometime. In order to make do with human shortcomings, the machine can be used to help people in their work. In this tactics the machine can play the following roles: 1) to sense accurate quantitative information which exceed human feeling range; 2) to supervise human actions and give advice to human or warn human when they make a mistake or in the dangerous situation; 3 ) to warn people by some means as soon as machine find human being in abnormal status because of fatigue, sleepiness, excessive drunk, and then to adjust human status by vibration, voice or other methods.

For example, during the automobile driving, the machine-aided-driving-system can help drivers make their vision broader, and correct drivers' errors. On the other hand, machine can monitor driver's status and remind the drivers ceasing drive during the abnormal status. So the driving security can be greatly improved.

\section{Machine Master/Human Slaver}

There are some systems with well-structured and good linearity can be controlled by machine intelligent system automatically, such as pilotless aircraft, automaton automobile, manless factory etc. But there were a few such systems successful and generally they cost much more.

Under these cases, the system performance can be improved based on the theory of HIS by the tactic of "Machine master/human slaver", which can be described as shown in figure 3 .

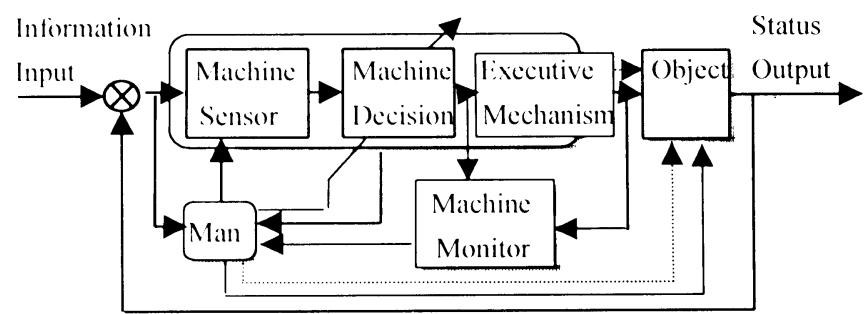

Figure 3 Machine Master / Man Slaver

In this kind of system, machine senses the circumstance's information automatically and makes decision with its knowledge base, then machine can do some work automatically. Man here can play the following roles: 1) to sense the qualitative information which machine senses difficulty; 2) to supervisor the machine's actions, and to correct what it is wrong immediately; 3) to extricate machine from difficult puzzled.
This tactic is often adopted in the simplicity task that is well-structure and good linearity, such as production line for singularity product, automatic steering the car under the simple circumstance, automatic driving the space shuttle in the outer space. In the control of these systems, man can introduce their own supervising advice in it, so as to improve the performance of these systems remarkably.

\section{Human Machine Cooperation}

There are many very complex questions in real life. All these questions will not be solved well only by man or only by machine. They should be solved by man and machine"s coordination. It would get the rational financial technology efficacy.

This tactic generally is divided into two methods, one is that the system acts by the result of man-machine's consultation; the another one is that the system acts by the result of real time simulation. These two methods can be described as figure 4 and figure 5 .

Figure 4 shows that man and machine sense the

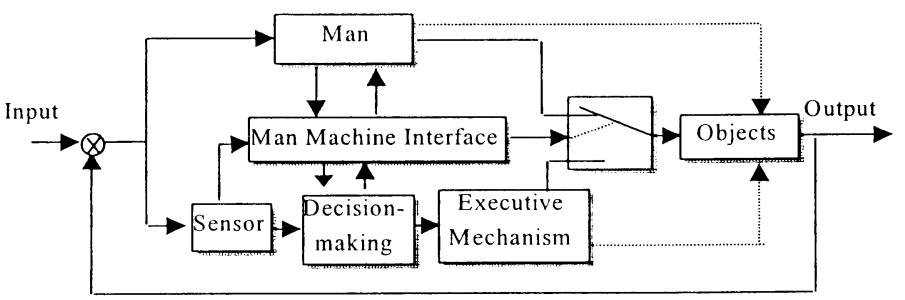

Figure 4 Man consult with machine in the "Man machine cooperation" tactic

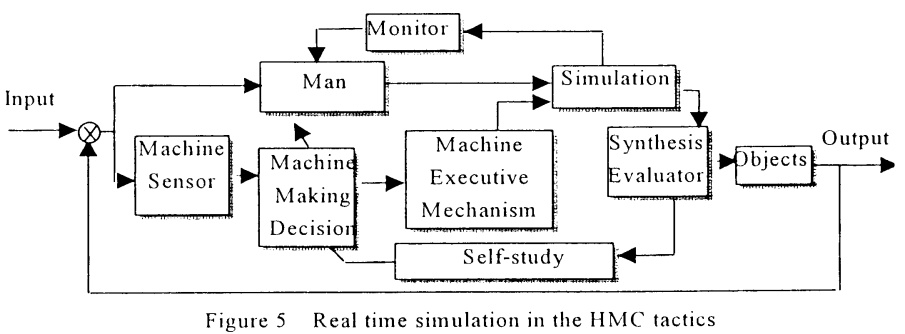

circumstance and make decision for the task together, when they all propose their own decisions and consult with each other. These consulting results will control the system. Tactic of the figure 5 shows that man and machine make decisions respectively by themselves according to their own perception and evaluation, then the decision will be made by the synthesis evaluator. The better decision will be applied in the system control. The machine will give man some signs or warnings when the synthesis evaluator find the output of machine is better, and the system will be controlled by the machine. In this 
case, machine will give man a clear description. On the contrary, the system will be controlled by the human when the synthesis evaluator find human's decision is better, and the machine knowledge base will be improved by a emulate algorithm. Therefore, the goals that man and machine learn from each other will be achieved.

\section{The Simulation of Humachine Intelligent Driving System}

An automobile normally meets very complex and driving circumstances on the road. The task of driving a car would be accomplished very well only by the means of the humachine intelligent driving methods in perceptive level, thinking level and executive level.

\section{1) Perceptive level}

During the period of driving a car, the driver must sense and deal with a vast amount of dynamic information such as the condition of the road, the other cars' speed and direction, the condition of his car and so on. Because of human sense threshold and limitation, the humachine sense must be realized in the perceptive level by many advanced sensors. For examples, People can help
Thinking level means decision-making level of a system. The drivers often make decisions depend on their experience, their skills or so. It is difficult for drivers to think all things over thoroughly when they meet the complex situations, and compared with computers, the drivers' computing speed is too slow. So the drivers must be helped by the machine. In the other way, people can drive a car by their intuitions which may be faster than the machine's computing and inference. Therefore the precise decision will be made by the humachine intelligent method.

\section{3) Executive Level}

Although the skilled drivers can drive the car well, they would make mistakes in the emergency because of their delay in reaction and delay in action. The machine aided manipulation device can be introduced in it to improve the security of the driving processes.

Therefore the model of the humachine intelligent driving system shown in figure 6 can be created based on the theory of HIS. The humachine intelligent car driving can be realized in perceptive, thinking and executive three level. The security and reliability of driving would be improved greatly by bringing human and machine's advantages together into full play in every level.

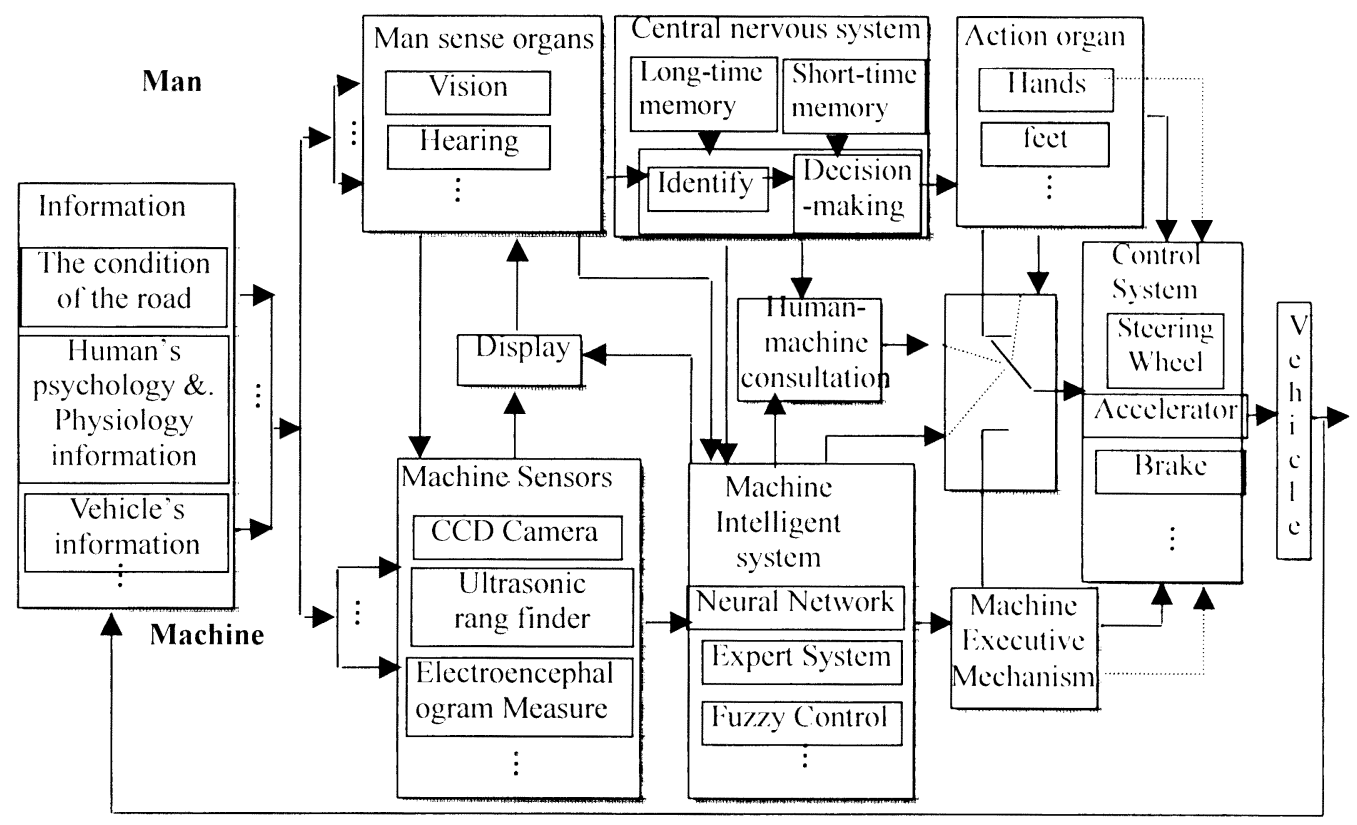

Figure 6 The Model of Humachined Intelligent I)riving System

machine identify the objects on the road which can drive over and which must be avoided. Machine can help people measure the width of road, the distance between the cars, the radius of the turn and so on.

2) Thinking level
The hardware platform shown in figure 7 and software platform in figure 8 were setup with a discarded jeep in order to fulfill humachine intelligent driving simulation experiments. In the simulation, the humachine intelligent system turns the test jeep in a corner, avoids obstruct, overtakes other cars and parks the jeep in a lane. 
All the tests are fulfilled on a virtual environment in computer. The experiments' results indicate that the

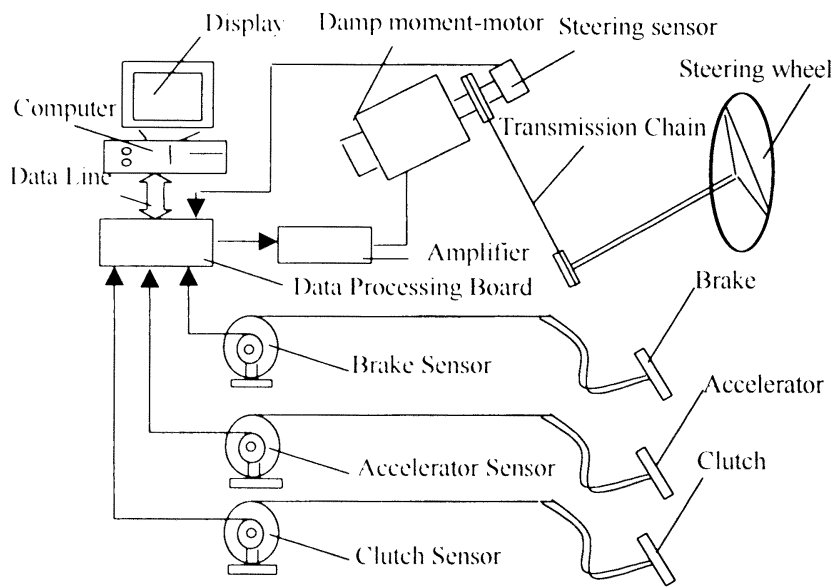

Figure 7 The llardware structure of the Driving Simulation device

effect of humachine intelligent automobile driving is better than driving by human alone or by machine only.

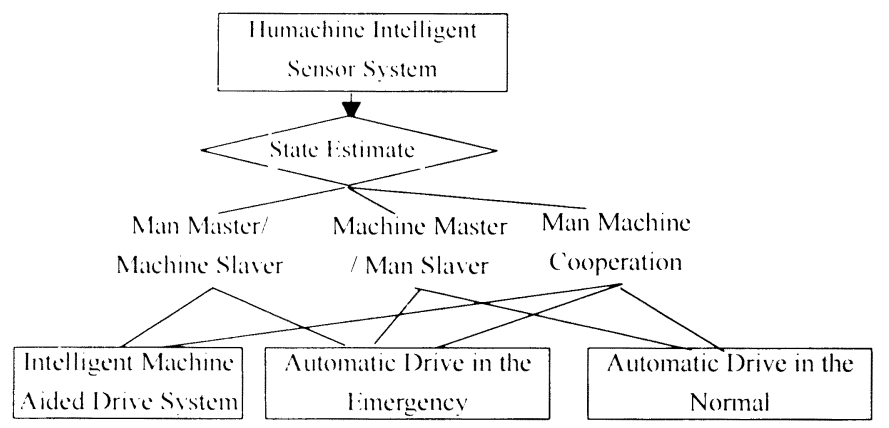

Figure 8 The software structure of the simulation experiments

\section{CONCLUSION}

In this paper, authors put forward the new method of setting up intelligent system; the model of the humachine intelligent system is discussed in details. The tactics of HIS' realization are described which are respectively named as "Human master / Machine slaver", "Machine master/Human slaver" and "Man Machine Cooperation". The experiments of humachine intelligent driving a car have been studied to implement the HIS theory. The results of these experiments indicate that the HIS theory is one of great methods to implement intelligent systems.

\section{REFERENCES}

1. Lu Yongxiang, Chen Ying. Foundation Of The Humachine System, Chinese Journal of Mechanical Engineering , 1994,30(6)

2. Lu Yongxiang, Chen Ying. Humachine-A New Word For The 21th Century, Chinese Journal of Mechanical Engineering, 1994,30(5)

3. Lu Yongxiang, Chen Ying. Academic Architecture And Key Techniques of The Humachine System, Chinese Journal of Mechanical Engineering, 1995,31(1)

4.Dai Ruwei. Metasyntactic Wisdom of Man Computer Cooperation, Pattern Recognition And Artificial Intelligence, 1994,7(3)

5.Thomas B. Sheridan, Telerobotics, Automation, and Human Supervisory Control, The MIT Press Cambridge, Massachusetts, London England, 1992

6.Mayr, R. Automated steering control for vehicles, Int. J. of Vehicle Design, 1995, Vol.16, No. 4/5

7.Yang Canjun. A Study on the Theory and Practice of Humachined Intelligent System, PHD. Dissertation . Zhejiang University, China. 1997

8.Abe M., A study on effects of roll moment distribution control in active suspension on improvement of limit performance of vehicle handing. Int. J. of Vehicle Design, 1994,Vol. 15 